\title{
Historia del concepto de dolor total y reflexiones sobre la humanización de la atención a pacientes terminales
}

\author{
History of the Concept of Total Pain \\ and Reflections on Humanization \\ of Assistance for Terminal Patients
}

\section{História do conceito de dor total e reflexões sobre a humanização da atenção a pacientes terminais}

Juan Rafael López Sánchez, MD*1.

Silvia Rivera-Largacha, PhD. ${ }^{2}$

Recibido: 17 de febrero de 2017 / Aceptado: 20 de febrero de 2018

Doi: http://dx.doi.org/10.12804/revistas.urosario.edu.co/revsalud/a.6773

Para citar este artículo: López-Sánchez JR, Rivera-Largacha S. Historia del concepto de dolor total y reflexiones sobre la humanización de la atención a pacientes terminales. Rev Cienc Salud. 2018;16(2):340-356. Doi: http://dx.doi.org/10.12804/revistas.urosario.edu.co/ revsalud/a.6773

\section{Resumen}

Introducción: en este artículo se estudia el concepto de dolor total con el objetivo de abrir una reflexión sobre los aportes teóricos y prácticos que esta categoría puede ofrecer en el cuidado de pacientes terminales, enfatizando en la necesidad de humanizar la atención, como modo de restituir la calidad de vida y la dignidad de los pacientes, sus familias y sus allegados, y como modo de dignificar y mejorar la calidad y las condiciones del trabajo de los profesionales clínicos que acompañan a este tipo de pacientes en su tránsito hacia la muerte. Desarrollo: a lo largo del artículo se analiza el proceso histórico y epistemológico del cual surge el término dolor total como categoría que aporta herramientas teóricas que permiten orientar el diagnóstico y el abordaje terapéutico del dolor en las condiciones particulares propias de la enfermedad terminal. Conclusión: el concepto de dolor total permite entender el proceso del final de la vida como una experiencia de enfermedad (disease), padecimiento (illness) y disfunción social (sickness) que comporta diferentes formas de sufrimiento que deben ser atendidas de manera integral. La atención integral significa que el profesional de la salud debe centrarse más en el paciente que en su patología, y ofrecerle una mejor calidad de vida y acompañamiento en el proceso de morir.

Palabras clave: historia, dolor, comunicación y humanización de la atención.

1 Universidad Distrital Francisco José de Caldas, Universidad Nacional de Colombia, Universidad del Salvador, Escuela de Medicina y Ciencias de la Salud Colegio Mayor de Nuestra Señora del Rosario, Hospital Universitario Mayor Mederi.

Autor de correspondencia: jrlsmd@hotmail.com

2 Escuela de Medicina y Ciencias de la Salud Colegio Mayor de Nuestra Señora del Rosario. silvia.rivera@urosario.edu.co 
Abstract

Introduction: In this paper we study the concept of total pain to open a reflection on the theoretical and practical contributions that this category can offer in terminal patients care, emphasizing in the need of humanize the attention, as a way to restore the quality of life and the dignity of the patients, their families, their friends and as way to dignify and improve the quality and the work conditions of the clinical professionals who keep company to these patients in their transit to death. Development: Through this paper we analyze the historical and epistemological process from which arises the term total pain as a category that provides theoretical tools that allow direct diagnosis and the therapeutic approach of pain in the particular conditions of the terminal illness. Conclusions: The concept of total pain allows to understand the process of end of life as an experience of disease, a condition (illness) and a social dysfunction (sickness) which involves different forms of suffering that may need to be treated in a holistic manner. Holistic care means that the health care professional should focus more on the patient, and not on its pathology, offering a better quality of life, and support in the process of dying.

Keywords: history, pain, communication and humanization of assistance.

\section{Resumo}

Introdução: neste artigo estuda-se o conceito de dor total com o objetivo de abrir uma reflexão sobre os aportes teóricos e práticas que esta categoria pode oferecer no cuidado de pacientes terminais, enfatizando na necessidade de humanizar a atenção, como modo de restituir a qualidade de vida e a dignidade dos pacientes, suas famílias e seus parentes, e como modo de dignificar e melhorar a qualidade e as condições do trabalho dos profissionais clínicos que acompanha a este tipo de pacientes em seu trânsito à morte. Desenvolvimento: ao longo do artigo se analisa o processo histórico e epistemológico do qual surge o termo dor total como categoria que aporta ferramentas teóricas que permitem orientar o diagnóstico e a abordagem terapêutica da dor nas condições particulares próprias da doença terminal. Conclusão: o conceito de dor total permite entender o processo de fim da vida como uma experiência de doença (disease), padecimento (illness) e disfunção social (sickness) que comporta diferentes formas de sofrimento que devem ser atendidas de maneira integral. A atenção integral significa que o profissional da saúde deve centrar-se mais no paciente que em sua patologia, e oferece-lhe uma melhor qualidade de vida, e acompanhamento no processo de morrer.

Palavras-chave: história, dor, comunicação e humanização da atenção.

\section{Introducción}

"El estudio del dolor conduce a una medicina humana en todos sus gestos" René Leriche (1)

El 18 de mayo de 2016 se presentó en la revista Pain la nueva definición de dolor aceptada dolor es una experiencia angustiosa asociada a un daño tisular real o potencial, con componentes sensoriales, emocionales, cognitivos y sociales”. Esta nueva definición propone integrar al estudio y a la atención clínica del dolor, elementos que han sido encontrados por la investigación en los últimos cincuenta años (2). La nueva definición es una revisión de la que había sido propuesta por la IAsP, siguiendo el trabajo de Merskey, y Bogduk en la que se 
señala el dolor como "una experiencia sensorial y emocional desagradable, asociada con un daño tisular, real o potencial, o descrita en términos de dicho daño" (3).

La nueva definición se plantea como más completa al describir el dolor como una experiencia angustiosa e inquietante. Además, recoge los componentes emocionales y sensoriales que ya habían sido reconocidos desde los años noventa por la IASP y resalta los aspectos cognitivos y sociales que hasta ahora habían estado ausentes de la definición y que proporcionan una dimensión más comprensiva y amplia del dolor.

De acuerdo con Williams y Craig, esta nueva definición tiene como objetivo promover una mejor investigación científica y un cuidado de la salud óptimo y generar el interés por desarrollar estrategias de investigación e intervención que permitan ir más allá de la queja sobre el dolor, que según afirman, es una característica política y retórica importante, pero distractora de la definición clásica del dolor (2). Igualmente, señalan la necesidad de reconocer el dolor como un fenómeno que tiene un valor funcional y adaptativo dentro de un amplio rango de comportamientos no verbales.

Macknik y Martinez-Conde explican cómo, desde las neurociencias, se han acumulado pruebas sobre el carácter emocional, cognitivo y social que tiene la experiencia del dolor, y que permite pensar en este más que como una experiencia sensorial, como una emoción socialmente compartida, enseñada y aprendida (de allí su carácter cognitivo) (4). Es decir, que aunque el dolor es, generalmente, el resultado de una afección física, la raíz primaria de este es la emoción individual y compartida que aparece ante la presencia real o potencial de un daño tisular. Esta experiencia, como cualquier emoción, está complejamente intrincada con las representaciones sociales y por la interiorización de estas representaciones que cada sujeto aprende y adopta con respecto al daño físico. De ahí, la gran diversidad y variabilidad que existe en la forma en que las personas perciben y afrontan el dolor. Esto lleva a pensar que, aunque el manejo del dolor puede abordarse desde una perspectiva orgánica, por ejemplo, con el uso de fármacos, con el control de la inflamación, con la inmovilización de los tejidos, entre otros, las herramientas de atención cognitiva, psicológica y social tienen una importancia central.

\section{Desarrollo}

【 a Organización Mundial de la Salud define los cuidados paliativos como un enfoque que mejora la calidad de vida de los pacientes y sus familias que enfrentan los problemas asociados a la enfermedad peligrosa para la vida, a través de la prevención y alivio del sufrimiento mediante la identificación temprana y acertada evaluación y tratamiento del dolor y otros problemas, físicos, psicosociales y espirituales (5). 
En este sentido, los cuidados paliativos se enfocan en

Proporcionar alivio para el dolor y otros síntomas angustiantes; afirmar el morir como un proceso normal y como parte de la vida; intentar no acelerar o aplazar la muerte; integrar los aspectos psicológicos y espirituales del cuidado del paciente; ofrecer un sistema de apoyo para ayudar a los pacientes vivir tan activamente como sea posible hasta la muerte; ofrece un sistema de apoyo para ayudar a la familia a enfrentar la enfermedad de los pacientes y su propio duelo; utilizar un enfoque de equipo para atender las necesidades de los pacientes y sus familias, incluyendo el asesoramiento del duelo, de ser necesario; mejorar la calidad de vida y si se puede, influir también positivamente en el curso de la enfermedad (5).

En el presente artículo se analiza el concepto de dolor total a la luz de la nueva definición propuesta por la IASP, a partir de la cual se establecen como centrales los elementos sociales y cognitivos que lo componen. Esta nueva definición permite tener una mayor conciencia de las múltiples dimensiones de la experiencia álgica y repensar las estrategias de humanización del acompañamiento al enfermo terminal, puesto que señala de manera más profunda cómo el manejo del dolor no solo se refiere al reconocimiento e intervención sobre sus manifestaciones orgánicas, sino al reconocimiento y acompañamiento del paciente y de su entorno en esta experiencia compleja de sufrimiento. Esta nueva definición permite entender que la experiencia de dolor no es independiente al estrés y otros problemas psicosociales asociados con la enfermedad y, en el caso de pacientes terminales, al proceso de muerte. Es decir, que el dolor y los otros problemas atendidos por los cuidados paliativos jamás se presentan de manera independiente el uno del otro. Por lo tanto, la atención integral al dolor, planteada desde el concepto de dolor total concibe el manejo del dolor como un trabajo de intervención que cubre también en parte la atención al estrés y otros problemas psicosociales asociados con la enfermedad y con el proceso de muerte, con el objetivo de mejorar la calidad de vida del paciente y su entorno más cercano.

Frecuentemente, el dolor está vinculado con el sufrimiento y la desesperanza, y es el acompañante casi inevitable del enfermo terminal. Cuando el dolor adquiere un carácter crónico y progresivo, altera el funcionamiento personal, familiar y social, lo que afecta, de manera directa, la calidad de vida del paciente y de su entorno. Según la IAsP, el dolor es una experiencia angustiosa, pero lo que no es claro es a quién corresponde la angustia. Se puede decir que emerge en quien padece en carne propia el dolor, pero también en sus allegados y en los profesionales de la salud que acompañan esta experiencia. El sufrimiento y la angustia emocional, espiritual y social que caracterizan la experiencia álgica, en especial en la enfermedad terminal, son elementos indisociables que forman parte de la experiencia compleja del dolor. Cuando este dolor se presenta en el caso de una enfermedad terminal, estas dimensiones deben ser analizadas y tomadas en cuenta en el tratamiento del paciente, dentro de lo que se denomina como "la experiencia de dolor total" $(5,6)$. 
En este artículo se revisa el proceso histórico y epistemológico a partir del cual surge el término dolor total como categoría que aporta herramientas teóricas y prácticas que permiten orientar el diagnóstico y el abordaje terapéutico del dolor y, con este, de otras dimensiones del cuidado en las condiciones particulares de la enfermedad terminal. Con esta revisión se pretende abrir una reflexión sobre los aportes teóricos y prácticos que ofrece esta categoría en el cuidado de pacientes terminales, enfatizando en la necesidad de humanizar la atención, como modo de restituir la calidad de vida y la dignidad humana del paciente, su familia, sus allegados y como modo de dignificar y mejorar la calidad y las condiciones del trabajo del personal clínico que acompaña a estos pacientes en su tránsito hacia la muerte.

\section{Historia del redescubrimiento del dolor}

En los años cincuenta y sesenta del siglo xx se dieron nuevos paradigmas morales, culturales, sociales y científicos; en este contexto se desarrolló una revolución en la explicación y tratamiento del dolor y de la medicina paliativa. Dentro de la aplicación actual del concepto de dolor total fueron John J. Bonica, Cicely Saunders y Elisabeth Kübler Ross quienes dieron aportes centrales para el desarrollo y la aplicación de esta noción en el cuidado de pacientes terminales.

Bonica nació en 1917 en Italia, se graduó como médico en New York en 1942, luego de terminar su entrenamiento militar en 1944, fue asignado como jefe de Anestesia del Centro de Operaciones de Madigan Army Hospital en Tacoma, Washington, donde se atendían los soldados heridos en la II guerra mundial (6). Bonica se interesó por aliviar el dolor a promover el uso de anestesia regional en muchos de los procedimientos quirúrgicos. Además, identificaba en sus colegas una gran reticencia en el uso de opiáceos, que según él no eran formulados a tiempo, ni en las dosis adecuadas, a causa del miedo a los fenómenos de dependencia y de tolerancia. Los años de la guerra marcaron su interés por el manejo del dolor y lo confrontaron con la escasez de recursos teóricos y terapéuticos para entender y aliviar los distintos tipos de dolor. Así, se interesa por entender el dolor como un fenómeno complejo que requería un estudio y un manejo multidisciplinario que incluyera el estudio de la influencia de la mente.

El resultado de su trabajo se consolida en 1953 con la publicación de su libro Tratamiento del dolor, con estudio especial del empleo del bloqueo analgésico en el diagnóstico, pronóstico $y$ terapéutica, considerado como el primer texto moderno de medicina del dolor y el punto inaugural de una nueva era de atención a la experiencia álgica. Bonica explica esta experiencia como un fenómeno dual compuesto por aspectos fisiológicos (percepción) y subjetivos (emoción) que incluían funciones cognitivas complejas (5, 7-9). Bonica recupera el interés público por el estudio y el manejo del dolor, teniendo en cuenta la subjetividad del paciente e introduce la expresión ‘clínica del dolor’ que, según Moscoso (10), dirige la atención hacia 
el esfuerzo de transformación del sufrimiento privado en un asunto de responsabilidad y un objeto de reflexión pública y de atención primaria.

Dentro de este contexto, Cicely Saunders, una enfermera y trabajadora social hospitalaria, influenciada entre otros por los trabajos de Bonica, crea en 1964 la categoría de dolor total y funda en 1967 el primer hospicio moderno para enfermos terminales (5, 7-9). El trabajo de Saunders iba en contra de la ortodoxia del trabajo clínico típico de la medicina de su tiempo. Desde su doble formación inicial, fomentó el interés por las emociones de los pacientes y por cómo estas interactuaban con la enfermedad. En el aspecto farmacológico de su trabajo, de manera empírica, empezó a ajustar las dosis de morfina que administraba a sus pacientes, según la intensidad de dolor. Finalmente, motivada por su experiencia con pacientes terminales en 1957, se graduó como médica y empezó a trabajar en el Departamento de Farmacología de la Escuela de Medicina del St. Mary’s Hospital, donde se dedicó a la investigación sobre el manejo y el alivio del dolor, haciendo énfasis en el acompañamiento emocional, la escucha y la atención a las necesidades particulares de los pacientes. Su trabajo permitió la humanización de la atención en las salas de pacientes terminales. En 1962 demostró ante la Royal Society of Medicine que no había adicción a opioides en pacientes terminales con dolor por cáncer $(7,8,10,11)$.

Las propuestas de Saunders emergen dentro de un contexto médico marcado por las propuestas de la nueva medicina social de John Ryle (1889-1950), quien señala la importancia de proponer una medicina en la que el papel del médico no deba ser encontrar la enfermedad en el cuerpo del paciente, sino "entender a cada enfermo en el contexto de la enfermedad" (10). El entender la enfermedad en contexto y dentro de la evolución de un ciclo natural, lleva a establecer parámetros de tratamiento ajustados a sanar, en caso de ser posible la cura, o a ofrecer medidas paliativas para quienes se dirigen inevitablemente hacia la muerte. Estas medidas son consideradas una forma de trato humanitario y de restitución de la dignidad, a partir de la disminución del dolor y el aseguramiento de la calidad de vida del paciente terminal.

Sumando los conocimientos adquiridos por años y en colaboración con Twycross y otros investigadores, Saunders publicó en 1963 el libro Oxford Text Book of Palliative Medicine, en donde afirma:

Creemos que hay pocas reglas en el tratamiento del dolor inevitable en la fase final. Lo primero es una adecuada evaluación de los síntomas [...] para tratar el dolor y todo lo que hace miserable al paciente [...] es claro que cada muerte es individual como lo fue la vida y que esa experiencia total se refleja en cada persona [...]. Por eso es necesario hablar del dolor total (12).

Otra investigadora central en la historia del estudio y el manejo del dolor y los cuidados paliativos es la psiquiatra Elisabeth Kübler-Ross, quien, siguiendo los aportes de Saunders 
en 1969, publica el libro Sobre la muerte y los moribundos (13). Este trabajo que subraya la necesidad de dirigir los servicios de salud al final de la vida, aboga por la implementación de una atención humanizada que tenga en cuenta que las personas murientes presentan cambios mentales y emocionales singulares. Cambios que describe en cinco etapas: la negación, la ira, la negociación, la depresión y la aceptación. Estas etapas son una herramienta descriptiva que permite entender el proceso del duelo en la mayoría de las personas, lo que permite una comprensión de los aspectos psicológicos más característicos de la confrontación con la muerte y ver la muerte como un episodio normal de la vida para guiar el acompañamiento de los pacientes y facilitar un trato humano y digno (13-16).

Además de estrategias dialógicas, Kübler-Ross rescata la importancia de los gestos físicos, como el tacto como modo de comunicación y de sostén emocional y espiritual para el enfermo: "al final el moribundo quiere una persona amada, sentada confortablemente y en silencio al lado de la cama, sin palabras, pero tocando las manos, masajeando los cabellos, dando suaves pellizcos en la pie, aplicando una crema o simplemente estando allí” (13).

El estar en contacto con los pacientes, tiene su propio lenguaje que humaniza, disminuye barreras, aproxima, comparte y comunica. Los médicos deben demostrar de manera verbal y no verbal que la parte más importante de la entrevista médica es el paciente que está frente a ellos y no la enfermedad $(13,17,18)$.

Los trabajos de Bónica, Saunders y Kübler Ross impulsan el examen crítico en lo que concierne la relación del personal de la salud y el paciente, que pretende recuperar el valor de las prácticas clínicas tradicionales que tenían en cuenta la subjetividad del paciente. Con esto resurge el interés por ofrecer una atención clínica atenta a la subjetividad del paciente con dolor o en proceso de morir.

Bonica, Saunders y Kübler Ross abren las puertas al desarrollo una práctica de atención centrada en el paciente al resaltar la importancia de entender social y fenomenológicamente el cuerpo, y abrir la puerta a la necesidad de investigar e intervenir sobre el dolor y otros problemas orgánicos y psicosociales, desde una perspectiva multidimensional que incluya sus componentes psicológicos e interaccionales que acompañan la enfermedad terminal. La recuperación de esta "sabiduría clínica clásica” se combina en los trabajos de Bónica y Saunders con el énfasis en la importancia de encontrar evidencia y credibilidad científica en el diseño de nuevos tratamientos, que permitieron un mejor manejo del dolor al usar herramientas farmacológicas que durante mucho tiempo fueron temidas, desestimadas o incluso satanizadas $(19,20)$. 


\section{Las dinámicas de la relación médico-paciente: puntos de introducción para el análisis de la humanización de la atención al paciente con dolor}

Para reflexionar sobre el impacto que históricamente ha tenido la labor de quienes han abierto el campo de la clínica del dolor y la humanización de la atención a los pacientes terminales, se propone una reflexión sobre las implicaciones de las representaciones de la enfermedad, del dolor y de las relaciones médico-paciente en el campo de la medicina del dolor y paliativa.

Desde los estudios sociales de las ciencias existen tres formas distintas de nombrar la experiencia de la enfermedad: enfermedad (disease), padecimiento (illness) y disfunción social (sickness) (21). Estas formas de describir la experiencia del enfermar han sido adoptadas además por los estudios de salud pública, especialmente, a partir del trabajo del epidemiólogo Susser en 1973 (22).

Se habla de padecimiento (illness) para referirse al sentimiento subjetivo propio de cada persona de sentir una afección que compromete su bienestar. El padecimiento no hace referencia a una patología particular, sino a la experiencia personal de molestia, malestar y agotamiento. El padecimiento está marcado por las representaciones sociales e históricas que nutren las representaciones que cada persona adopta con respecto a su propio sufrimiento. Así, aunque la experiencia de cada enfermo es particular, está siempre impregnada por las representaciones que adopta de su entorno y de la historia que lo precede. De allí la importancia de la dimensión sociocultural e histórica del padecimiento. Esta dimensión es caracterizada por el término ‘sickness’ que habitualmente se traduce como 'disfunción social' y que hace referencia a las concepciones sociales y culturales sobre la salud. El cáncer, por ejemplo, en las sociedades occidentalizadas es representado — de acuerdo con Sontag — como una tragedia, una amenaza bélica que ataca el cuerpo y frente al que hay que dar la batalla (23). Las representaciones sociales de la experiencia de enfermar influencian la manera en que el enfermo reacciona a su situación. Las percepciones y las representaciones sociales de la enfermedad pueden modificar la manera como el enfermo percibe, asume y reacciona frente a sus síntomas (23-25).

El término enfermedad (disease) da cuenta del proceso de comunicación y de transformación que tiene la situación del enfermar, cuando el padecimiento del enfermo está vinculado a una narrativa social propia de la medicina. El modelo biomédico focaliza su atención en el proceso patológico para lograr un diagnóstico, seguido de un tratamiento cuyo objetivo es restaurar la integridad y la funcionalidad fisiológica del paciente. El proceso patológico, que puede o no producir síntomas, resulta en la enfermedad del paciente. Puede existir, por ejemplo, una enfermedad que no necesariamente sea un padecimiento (un tumor cerebral descubierto accidentalmente por una imagen diagnóstica, que nunca generó síntomas). Puede también darse un padecimiento (illness) sin que exista necesariamente una enfermedad (disease), es el caso de la hipocondría (21). 
El modelo de la biomedicina entiende la enfermedad (disease) como un fenómeno de desequilibrio reconocido en virtud de la comprobación o la suposición de un origen orgánico (anatómico, fisiológico, etiológico o genético). El diagnóstico consiste en un reconocimiento y una aplicación de una categoría generalizadora a un patrón de signos y síntomas que pueden ser explicados (al menos parcialmente) por el funcionamiento anormal de la estructura, las funciones de las células, los tejidos, los órganos o sistemas del cuerpo. El tratamiento consiste en estrategias que tienen una base de comprensión empírica y racional de la enfermedad y que pretenden contrarrestar o hacer desaparecer (a partir de una técnica también empírica y racional) el desequilibrio o las lesiones que esta produce en el organismo (21).

Al pensar el dolor en esta triada de términos que nombran la experiencia de enfermar (padecimiento, enfermedad y disfunción social), se puede decir que el dolor en tanto que síntoma que acompaña la experiencia de enfermar atraviesa estas tres categorías. El dolor hace parte del padecimiento, puesto que es una experiencia sensorial y emocional angustiosa que produce un sufrimiento en la persona. El dolor es una de las primeras señales por medio de las que se manifiesta la enfermedad y es una de las experiencias más fuertemente asociadas con el padecimiento y de más difícil control (24-26).

Aunque la experiencia del dolor es particular, está siempre influenciada por las representaciones que adopta de su entorno quien lo padece y de la historia que lo precede. Así, el dolor tiene una dimensión sociocultural e histórica y hace parte de la experiencia de enfermar en tanto que 'disfunción social' $(10,24)$. En efecto, existen concepciones sobre el dolor que son constituidas social y culturalmente: la forma de soportarlo, de manejarlo, de obtener alivio, de luchar contra este, de sufrirlo o de reivindicarlo. Por ejemplo, son frecuentes las concepciones religiosas del dolor: pacientes que rechazan los tratamientos contra el dolor por considerarlos un impedimento para aprovechar su dolor como una forma de sacrificio a Dios, que consideran útil para obtener la salud física o la salvación espiritual (10, 25, 26).

El dolor, finalmente, puede hacer parte de la enfermedad (disease), porque quien lo padece, generalmente, trata de comunicar su experiencia para encontrar una solución. Cuando esta ayuda se busca en el sistema de salud, se establece un proceso de comunicación en el que el sujeto intenta vincular su padecimiento a la narrativa propuesta por las ciencias de la salud (27). El médico intenta identificar el proceso patológico de origen del dolor para tratarlo y restaurar la integridad y la funcionalidad fisiológica del paciente y eliminar este síntoma.

En los casos en los que el dolor persiste y se instala como una condición permanente (dolor crónico), este se convierte en una enfermedad en sí mismo. Dentro de la medicina del dolor, en ambos casos, cuando el dolor es un síntoma o una enfermedad, las estrategias de intervención quirúrgicas, biomédicas, farmacológicas e incluso psicoterapéuticas son cada vez más amplias y efectivas. Sin embargo, existe también una gran cantidad de padecimientos 
cuyo origen es aún desconocido y hay otros padecimientos cuyo tratamiento efectivo es aún inalcanzable para la medicina del dolor (20).

Finalmente, dentro de la naturaleza amplia del dolor habría que reconocer que este es también una señal de 'salud' como correcto funcionamiento orgánico. En este sentido, el dolor es la señal que alerta sobre cambios radicales y necesarios del cuerpo. Puede ser este el caso de los dolores de crecimiento, que hacen evidente un proceso de transformación física en niños y adolescentes; o es el caso de los dolores del parto que tienen una correlación con cambios fisiológicos y anatómicos que permiten la adaptación del cuerpo para el alumbramiento (28).

Dentro de estas tres formas de ver la enfermedad y el dolor (padecimiento, enfermedad y disfunción social), se pueden reconocer formas y momentos distintos de la relación entre médico y paciente en lo que concierne la comprensión y el manejo del dolor. En la relación médico-paciente se pone en juego la condición de una persona que padece una dolencia. El enfermo se encuentra aquejado por el dolor, la molestia y las diferentes sensaciones que evidencian un desequilibrio. Quien padece esta experiencia se encuentra dominado por lo incontrolable y lo incomprensible de su condición. A su vez, muchos afectos como la soledad, la angustia, la tristeza o, por el contrario, la aceptación y la paciencia pueden acompañar esta experiencia. Es a esta experiencia del enfermar que hace referencia el término illness, el padecimiento $(21,29)$.

Cuando el médico y el personal de la salud ocupan el lugar de aquel que intenta recibir el sufrimiento por medio de un logos, esta experiencia subjetiva propia al sujeto tiene un espacio de reconocimiento por parte de otro, que intenta darle forma y procura delimitar estas sensaciones desbordantes que generan un padecimiento. Es, a partir del reconocimiento de esta experiencia por parte de un otro diferente al enfermo, conocedor de los procesos patológicos del organismo, capaz de hacer un diagnóstico, proponer una terapéutica y ejecutarla, que el padecimiento del dolor se hace síntoma o enfermedad medicamente reconocida (disease).

El encuentro entre el médico o el profesional de la salud y el paciente está marcado de entrada por la incomprensión, pero a la vez por el acuerdo mutuo por intentar entender el origen y las posibles soluciones para el padecimiento. En este encuentro se establece una comunicación, en donde la identificación de signos por parte del clínico hace posible darle sentido a la queja del enfermo con respecto al dolor. La comunicación entre ambos permite resignificar y reorientar el sufrimiento del enfermo.

Desde el nacimiento de la medicina hipocrático galénica, el objetivo de la presencia del médico es poder encontrar el origen y el sentido de ese padecimiento, encontrar un logos que lo explique y que, en lo posible, permita intervenir sobre el sufrimiento (30). De tal forma, el encuentro entre el médico y el enfermo es inicialmente una experiencia sensorial, donde el médico está atento a los signos que se hacen evidentes en el paciente. En un primer 
momento, el enfermo no sabe nada sobre estos signos, carece de referentes para entender su condición y solo sufre por la dolencia percibida. El proceso de diagnóstico, de proposición de un tratamiento y su ejecución consolidan la relación entre el médico y el enfermo, con todos los matices que pueden existir en esta.

Ahora bien, la relación médico-enfermo está inmersa en una serie de particularidades sociales y psicológicas que caracterizan a ambos actores como individuos y actores sociales. La edad de cada uno, su sexo, su raza, su nivel educativo, el tipo de experiencia frente a la enfermedad padecida, entre otros, son factores que van a determinar la calidad y la dirección que toma el encuentro. Pero también en la relación entre médico y enfermo hay un contexto cultural y social en el que se da esta relación. Es este contexto al que ya se ha hecho referencia como disfunción social (sickness) (21). En primer lugar, las representaciones sociales de la enfermedad cumplen un papel importante en la experiencia del padecimiento y en la identificación de la enfermedad. Ambos, enfermo y médico, son sujetos sociales marcados por las representaciones de la enfermedad y de dolor, propias de su contexto. En segundo lugar, el contexto social y cultural en el que se da este encuentro está conformado por terceros actores que intervienen en la relación médico-enfermo. Estos terceros son el personal de salud (paramédicos, psicólogos, trabajadores sociales, etc.), personal administrativo (entes financiadores y aseguradores de los servicios de salud) y actores relacionales (el entorno afectivo del enfermo: familiares, amigos, asesores psicológicos o espirituales) $(26,27)$.

\section{El concepto de dolor total y el acompañamiento al enfermo terminal}

En 1964, Cicely Saunders desarrolla el concepto de dolor total recogiendo la experiencia del trabajo con pacientes terminales, en cuyas historias identifica vivencias de sufrimiento físico, mental, social, emocional y espiritual $(7-9,11)$. Al proponer este término, Saunders plantea un estilo de trabajo que pone en el centro del quehacer del clínico la subjetividad y la particularidad del paciente que padece dolor, en especial, si este dolor acompaña su tránsito hacia la muerte $(11,31)$. Para que el dolor no se convierta en el centro de la vida del paciente, el clínico debe poner en el centro de su atención a la persona. En el caso del paciente con cáncer avanzado, en algunos casos de sida y en la fase terminal de otras enfermedades, este concepto describe el dolor como un fenómeno complejo, en donde es importante identificar la experiencia del enfermo desde el concepto de 'sufrimiento' que, de acuerdo con Saunders, es una experiencia que "le sucede a las personas y no a los cuerpos". El sufrimiento es una categoría que permite redimensionar el dolor en múltiples aspectos:

Dolor físico: se hace presente en la experiencia sensorial de percepción de un daño tisular real o potencial. Los pacientes con cáncer presentan dolor en el $60 \%$ de los casos cuando se encuentran en tratamiento activo, en enfermedad avanzada en un $65 \%$, en fases finales o 
terminales de la enfermedad un $90 \%$ y en sobrevivientes de esta enfermedad en un $33 \%$. El 70\% de los pacientes relaciona de forma directa que el dolor no controlado empeora su calidad de vida, sumado a otros síntomas que pueden estar relacionados con efectos adversos de los tratamientos o de su patología en sí, como el insomnio, la fatiga crónica, la ausencia de apetito sexual, entre otros.

Dolor social: se evidencia en la preocupación del paciente por su familia, finanzas, pérdida de trabajo prestigio laboral, posición social perdida del rol familiar y por ende miedo al abandono y aislamiento.

Dolor psicológico: se presenta por enojo por fracasos terapéuticos, retraso en los diagnósticos, alteración de la imagen corporal, ineficiencia y dificultades de acceso a los servicios de salud, miedo al dolor a la muerte y sentimiento de desesperanza, que viven todos los pacientes independientes de la etapa de su patología.

Dolor espiritual: se produce por los interrogantes que el paciente puede llegar a hacerse independiente de si tiene o no una creencia religiosa o del tipo de doctrina religiosa con la que se identifique: ¿Por qué me pasa esto a mí? ¿Por qué Dios permite que esto me suceda? ¿Seré perdonado por mis faltas previas? ¿Me lo merezco como castigo a mis actos? La espiritualidad es diferente a religiosidad, los principios de los cuidados paliativos reconocen una dimensión espiritual o trascendente que deriva del impulso humano intrínseco de encontrar un significado y un deseo casi universal de lograr un cierto sentido a la propia vida en relación con otras personas y el mundo. Esta engloba los aspectos de la experiencia de cada persona, de sus creencias y de sus relaciones que tienen una importancia inherente y una conexión sentida con algo mayor en lo que se puede creer (27,31-33).

Adicionalmente, se han identificado cuatro factores puntuales que aumentan el riesgo de sufrimiento en el paciente; la incertidumbre o la falta de certeza sobre lo que sucederá en el futuro; sentimientos negativos como miedo, tristeza o ansiedad; la sensación de pérdida de control, y la amenaza a la autoestima como consecuencia de los cambios generados por el tratamiento que alteran la autoimagen y producen la pérdida de la autonomía (12).

El dolor crea sufrimiento y su presencia actúa como un estresante permanente que recuerda al enfermo su condición y no solo le abruma por el sufrimiento inmediato que le causa, sino también por la sensación de amenaza e impotencia sobre su futuro, particularmente cuando se acerca al peor dolor o cuando el dolor señala una inminencia de la muerte, de un daño orgánico grave o de una afección que pueda llevar a la invalidez.

A pesar de que desde los años cincuenta del siglo xx muchos expertos han reivindicado la necesidad de estudiar y atender el dolor de manera amplia, estos conceptos y recomendaciones clínicas no han sido incorporados de manera amplia en todos los escenarios de la atención clínica. Generalmente, se prioriza el manejo del dolor físico, dejando de lado los otros aspectos del dolor total, limitando las herramientas de comprensión del fenómeno, y restringiendo las posibilidades de manejo de los pacientes aquejados por esta experiencia. 
Para lograr un adecuado manejo del dolor es necesario lograr una mejor preparación en el personal de salud y una voluntad política general de las instituciones para cambiar la cultura en torno a la comprensión y la atención del dolor. La educación médica debe implementar niveles y estándares formativos tanto en pregrado como en posgrado en relación con el dolor. El tratamiento inadecuado del dolor es evitable en la mayoría de las circunstancias y pese a ello persiste, de forma generalizada, en gran parte a la no existencia de profesionales formados en su manejo (34).

Un manejo integral al paciente en todos los aspectos que encierra el dolor total, incluyendo el sufrimiento, implica que se hagan esfuerzos para que el personal clínico logre una mejor comunicación con los pacientes, lo cual exige una escucha activa que permita realizar un trabajo más humano y recuperar en el trabajo del personal clínico los aspectos subjetivos de la condición del paciente. Se trata de desarrollar un trabajo en donde el paciente sea visto como sujeto de derechos y no como un objeto de tratamiento $(33,34)$.

En el complejo entramado que constituye la relación médico-paciente, es importante reflexionar sobre el papel decisivo que tiene este espacio de la relación clínica, dentro de la generalidad del proceso de atención al paciente que sufren de dolor y, particularmente, aquellos para quienes el dolor se presenta en el proceso de deterioro generado por una enfermedad terminal. Por tratarse de una experiencia subjetiva y socialmente determinada, el diagnóstico y el tratamiento del dolor es un desafío para el personal clínico. La calidad, la asertividad del diagnóstico y tratamiento del dolor tienen una fuerte relación con la humanización de la actividad clínica $(35,36)$.

Sobre este tema, Haque y Waytz, retomado los términos de Gray, Gray y Wegner han señalado que la deshumanización se presenta dentro del campo de la salud cuando en todos o en parte de los componentes que integran la atención al paciente se presenta una negación o negligencia con respecto a la existencia de lo que en psicología se ha llamado "mente” (36). De acuerdo con estos autores, la noción de mente corresponde a la existencia de dos dimensiones esenciales: la capacidad de un sujeto de sentir dolor y placer, descrita por el término 'experiencia', y la capacidad de este de planear, intentar ejercer y finalmente ejercer una elección. De tal forma, la humanización en el trato correspondería al cuidado y a la atención minuciosa en estas dos dimensiones. La clínica del dolor y, dentro de esta, el concepto de dolor total es entonces una herramienta fundamental dentro de la humanización del trato al paciente que se enfrenta a una enfermedad terminal (36). Este concepto busca atender, de manera meticulosa y asertiva, la experiencia de cada paciente, es decir, las condiciones de dolor que aquejan a cada persona de acuerdo con sus características particulares (físicas y psicológicas), al estado en el que se encuentra en el desarrollo de la enfermedad, al contexto social que lo rodea y a la dimensión espiritual desde la cual vive su enfermedad y su enfrentamiento con la posibilidad de una muerte próxima. De igual forma, este concepto otorga las herramientas para dirigir el trabajo clínico hacia una reivindicación de la agencia del 
paciente, puesto que dentro de las directrices de trabajo que se plantean, se busca ofrecer a la persona múltiples posibilidades terapéuticas que tengan en cuenta su capacidad de decisión para elegir el camino que quiere tomar con respecto al rumbo de su tratamiento y a las condiciones que quiere tener en el proceso de enfrentarse la experiencia física, psicológica, social y espiritual de una enfermedad terminal y del dolor que la acompaña.

\section{Conclusiones}

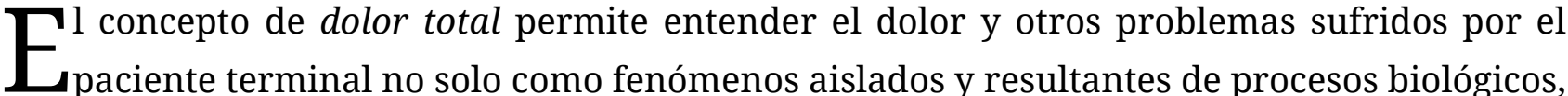
sino como realidades indisociables la una de la otra, que tienen orígenes e implicaciones diversas en las esferas sociales, psicológicas y espirituales del paciente. En este sentido, el concepto de dolor total permite entender la enfermedad y el proceso final de la vida como experiencias de enfermedad (disease), padecimiento (illness) y disfunción social (sickness) que comporta diferentes formas de sufrimiento que deben ser atendidas de manera integral. La mirada que acuña el concepto de dolor total permite al profesional entender la enfermedad terminal y sus problemas físicos, psicosociales y espirituales como un fenómeno múltiple en donde cada acto clínico no solo tiene implicaciones biológicas sino que se trata de un acto de comunicación verbal o no verbal que debe estar dirigido a mejorar la calidad de vida del paciente dentro de las diferentes esferas de su vida. Este concepto permite al profesional de la salud centrarse más en el paciente que el su patología, y ofrecerle una mejor calidad de vida, sin importar su pronóstico, esto significa acompañar al paciente a morir en paz y a vivir tan activamente como sea posible hasta el momento final. Al tener en cuenta las tensiones y el sufrimiento por las que atraviesa el paciente terminal, es posible acompañarlo y facilitarle la aceptación de la muerte como un proceso normal y natural de la vida. En los cuidados paliativos, la unidad de tratamiento es el paciente y sus allegados y, de manera tangencial, el medio social que los rodea. Al guiar el cuidado por la noción de dolor total, el personal clínico se centra más en la sanación que en la curación, lo que integra al paciente de manera social, psicológica y espiritual como un todo y permite un análisis más completo de su condición, generándole bienestar y alivio, sin acelerar el proceso de muerte y sin posponerla.

Según Saunders, "no se puede morir curado, pero sí se puede morir sanado" (32). Para esta pionera, la sanación es el proceso por el cual se alcanza una relación apropiada con uno mismo, con los demás y con Dios (o con el ser o la entidad superior en la que se crea). Sanar es entonces un proceso que permite una forma de equilibrio subjetivo, en donde se le restituye la dignidad humana al enfermo. Dentro de un sistema de salud fragmentado, y muchas veces dominado por el afán de curar los órganos o sistemas del cuerpo que producen 
las enfermedades, se requiere de un gran esfuerzo de parte de cada profesional de la salud y de parte de todo el sistema de atención en salud, para aprender a sanar a los pacientes que llegan al final de la vida. Sanar a estos pacientes implica restituir su dignidad humana y procurarles la maydor calidad de vida posible. Esta posición frente a la enfermedad y a la muerte, esta renuncia al furor curandis exige del profesional de la salud un reaprendizaje y una redefinición de su trabajo, en donde se abra espacio a la renuncia por el acto sobre-estimado de salvar la vida como único fin de la atención en salud(37). Esta renuncia y esta capacidad de redirigir el objetivo del profesional al acompañamiento del paciente terminal dignifican y humanizan el trabajo del profesional de la salud, lo que le da un profundo sentido a su quehacer cotidiano y al papel que ocupa hoy en día en la sociedad, el concepto de dolor total es entonces una herramienta fundamental dentro de la humanización del trato al paciente que se enfrenta a una enfermedad terminal. (38)

\section{Referencias}

1. Leriche R, De la santé à la maladie, la douleur dans les maladies, où va la médecine? Encyclopédie française, VI Paris: Librairie Aristide Quillet. 1936.

2. Williamsa A, Craig KD. Updating the definition of pain. Pain. 2016; 157(11):2420-3. doi: 10.1097/j.pain.0000000000000613

3. Merskey H, Bogduk, N. Part III: Pain Terms, A Current List with Definitions and Notes on Usage, Classification of Chronic Pain, IASP Task Force on Taxonomy $2^{\text {a }}$ Edition,. Seattle: IASP Press; 1994.

4. Macknik S, Martinez-Conde S, No Brain, No Pain, Pain is an emotion. Sci Am Mind. 2016;25(3). doi:10.1038/scientificamericanmind0716-1

5. Graham F, Clark D. Introduction, The changing model of palliative care, Medicine. 2007;36:64-6. doi: 10.1016/j.mpmed.2007.11.009

6. Álvarez T. Aproximación al tratamiento del dolor. Experiencia inicial en Antioquia. Rev ACED 2010;5(3):32-9.

7. Meldrum ML. A capsule history of pain management. JAMA. 2003;290(18):2470-5. doi:10.1001/jama.290.18.2470

8. Clark D. The history of cancer pain relief, J Pain Symptom Manage. 2005; V29(1):1. doi:10.1016/j.jpainsymman.2004.11.001

9. Clark D. Total Pain, disciplinary power and the body in the work of Cicely Saunders, 1958-1967. Soc Sci Med. 1999 sep; 49(6):727-36. doi: 10.1016/S0277-9536(99)00098-2

10. Moscoso J. Historia cultural del dolor. Madrid: Ed. Taurus; 2011.

11. Richmond C. Dame Cicely Saunders, BMJ, 2005 23;331(7510):238.

12. Rajagopol MR, Twycross R. Providing palliative care in resource-poor countries. In: Hanks G, Cherny NI, Christakis NA, Fallon M, Kaasa S, Porteno RK, editors. Oxford Textbook of Palliative Medicine. 4a ed. Oxford: Oxford University Press; 2010. p. 23-37.

13. Kübler Roos E. Sobre la muerte y los moribundos. Madrid: Ed. Debolsillo; 2013. 
14. Kübler Roos E. La Rueda de la vida 2ª edición. Barcelona: Zeta; 2011.

15. Kübler Roos E. La muerte un amanecer 24ª edición. Barcelona: Luciérnaga; 2011.

16. Montes de Oca Lomeli GA. Historia de los cuidados paliativos. Rev Dig Uni. 2006;7(4):1-9.

17. Pérez T, Castañeda J. Aspectos bioéticos en el manejo de dolor por cáncer. Rev Bio. 2010 ago;9-14.

18. Álvarez T. La magia de los últimos días. Rev ACED. 2010;5(3):57-64.

19. Seymour J. Clark D, Winslow M, Pain and Palliative Care: The Emergence of New Specialties, Journal of Pain and Symptom Management, 2005;29(1):2-13.

20. Caraceni A1, Hanks G, Kaasa S, Bennett MI, Brunelli C, Cherny N, et al. Use of opioid analgesics in the treatment of cancer pain: evidence-based recommendations from the eapc. Lancet Oncol. 2012;13(2):e58-68. doi: 10.1016/S1470-2045(12)70040-2

21. Leder D. Health and Disease. V. The Experience of Health and Illness. En Post S G. (ed.). Encyclopedia of Bioethics. New York: Macmillan Reference; 2004. p. 1081-7.

22. Susser MW. Causal Thinking in the Health Sciences: Concepts and Strategies in Epidemiology Oxford: Oxford University Press; 1973.

23. Sontag S. La enfermedad y sus metáforas y El sida y sus metáforas. Trad. de Mario Muchnik. Madrid: Debolsillo; 1996.

24. Péoc'h N. Représentations sociales de la douleur, Les dossiers des sciences de l'éducation [internet]. 2014 [citado 2016 dic 02]; 31. Disponible en: http://dse.revues.org/593; doi: $10.4000 /$ dse.593

25. Le Breton D. Antropología del dolor. Barcelona: Seix Barral; 1999.

26. DelVecchio MJ, Brodwin P. Pain as Human Experience: An Anthropological Perspective (Comparative Studies of Health Systems and Medical Care). California: Univ of California Press; 1994.

27. Zborowski M. People in pain. San Francisco: Jossey-Bass; 1969.

28. Lowe NK. The nature of labor pain. Am J Obstet Gynecol. . 2002;186(5 Suppl Nature):S16-24. doi:10.1067/mob.2002.121427

29. Aldricha S, Ecclestonb Ch. Making sense of everyday pain. Soc Sci Med. 2000;50(11):1631-41.

30. Bynum W. Medicine at the Bedside. The History of Medicine: A Very Short Introduction. Oxford: Oxford University Press; 2008.

31. De Simone G, Tripodoro V. Fundamentos de Cuidados Paliativos y control de Síntomas. Buenos Aires: Pallium Latinoamérica; 2006.

32. Bruera E. Palliative Care. Nature Reviews. 2013;10(2):108-11.

33. Astudillo W, Mendenueta C. Principios básicos para el control del dolor total. Rev. Soc. Esp. 1999;6(1):29-40.

34. Mezei L, Murinson BB. Johns Hopkins Pain Curriculum Development Team. Pain Education in North American Medical Schools. J Pain. 2011;12(12):1199-208. doi: 10.1016/j. jpain.2011.06.006

35. Quevedo E. Comprensión histórico-crítica del proceso salud-enfermedad: base para una renovación curricular en medicina. Educación Médica. Diseño e implementación de un currículo basado en resultados del aprendizaje. Bogotá: Editorial Universidad del Rosario; 2012. 
36. Sultan O, Waytz A. Dehumanization in Medicine: Causes, Solutions, and Functions. Per Psy Sci. 2012;7(2)176-86 doi: 10.1177/1745691611429706

37. Gray HG, Gray K, Wegner D. Dimensions of Mind Perception, Science. 2007;315:619.

38. Johnson S, Clayton J, Butow PN, Silvester W, Detering K, Hall J, et al. Advance care planning in patients with incurable cancer: study protocol for a randomized controlled trial. BMJ Open. 2016; 6(12):e012387. doi: 10.1136/bmjopen-2016-012387 